Article

\title{
Aberrant Glycosylation Augments the Immuno-Stimulatory Activities of Soluble Calreticulin
}

\author{
Fang-Yuan Gong ${ }^{*}{ }^{\dagger}$, Zheng Gong ${ }^{\dagger}$, Cui-Cui Duo, Jun Wang, Chao Hong and Xiao-Ming Gao * \\ Institute of Biology and Medical Sciences, Soochow University, Suzhou 215123, Jiangsu Province, China; \\ 20154221101@stu.suda.edu.cn (Z.G.); gfy0308@sina.com (C.-C.D.); jwang79@suda.edu.cn (J.W.) \\ chaohong@suda.edu.cn (C.H.) \\ * Correspondence: gongfangyuan@suda.edu.cn (F.-Y.G.); xmgao@suda.edu.cn (X.-M.G.); \\ Tel.: +512-65881547 (F.-Y.G.); +512-65881256 (X.-M.G.) \\ + These two authors contribute equally to this work.
}

Received: 31 January 2018; Accepted: 23 February 2018; Published: 27 February 2018

\begin{abstract}
Calreticulin (CRT), a luminal resident calcium-binding glycoprotein of the cell, is a tumor-associated antigen involved in tumorigenesis and also an autoantigen targeted by autoantibodies found in patients with various autoimmune diseases. We have previously shown that prokaryotically expressed recombinant murine CRT (rCRT) exhibits strong stimulatory activities against monocytes/macrophages in vitro and potent immunogenicity in vivo, which is partially attributable to self-oligomerization of soluble rCRT. However, even in oligomerized form native CRT (nCRT) isolated from mouse liver is much less active than rCRT, arguing against the possibility that self-oligomerization alone would license potent pro-inflammatory properties to nCRT. Since rCRT differs from nCRT in its lack of glycosylation, we wondered if aberrant glycosylation of eukaryotically expressed CRT (eCRT) would significantly enhance its immunological activity. In the present study, tunicamycin, an $\mathrm{N}$-glycosyltransferase inhibitor, was employed to treat $\mathrm{CHO}$ cells (CHO-CRT) stably expressing full-length recombinant mouse CRT in secreted form for preparation of aberrantly glycosylated eCRT (tun-eCRT). Our biochemical and immunological analysis results indicate that eCRT produced by CHO-CRT cells is similar to nCRT in terms of glycosylation level, lack of self-oligomerization, relatively poor immunogenicity and weak macrophage-stimulatory activity, while tun-eCRT shows reduced glycosylation yet much enhanced ability to elicit specific humoral responses in mice and TNF- $\alpha$ and nitric oxide production by macrophages in vitro. Given that abberant glycosylation of proteins is a hallmark of cancer cells and also related to the development of autoimmune disorders in humans, our data may provide useful clues for better understanding of potentiating roles of dysregulated glycosylation of molecules such as CRT in tumorigenesis and autoimmunity.
\end{abstract}

Keywords: calreticulin eukaryotic; macrophage; glycosylation

\section{Introduction}

Calreticulin (CRT) is an endoplasmic reticulum (ER) residential glycoprotein playing important roles in maintaining intracellular calcium homeostasis and also facilitating correct folding of major histocompatibility complex (MHC) class I molecules [1,2]. It is also regarded as a tumor-associated antigen because CRT is present on the membrane surface of various tumor cells and soluble CRT can be detected in the body fluid of patients with lung or bladder cancer [1-4]. The N-domain of CRT has been labeled as "vasostatin" for its ability to facilitate blood vessel formation in cancerous tissues [5]. Additionally, CRT is an important autoantigen targeted by autoantibodies in patients with 
autoimmune disorders such as rheumatoid arthritis (RA) and systemic lupus erythematosus (SLE) [3]. The angiogenesis activity of CRT may contribute to the joint inflammation of RA [6]. However, detailed molecular mechanisms for roles of CRT in tumorigenesis and autoimmunity so far remain illusive.

Our earlier studies showed that prokaryotically expressed recombinant murine CRT (rCRT, without the leader and ER-retention sequences) and CRT fragment covering amino acid residues 39-272 (rCRT/39-272, including the N and partial P domains) are capable of inducing strong antibody responses in mice and also macrophage production of tumor necrosis factor- $\alpha$ (TNF- $\alpha$ ) and interleukin (IL)- 6 in vitro $[7,8]$. Since TNF- $\alpha$ and IL-6 represent predominant inflammatory cytokines in the pathogenesis of RA and SLE $[9,10]$, our results underlie a strong correlation between serum CRT levels and autoimmune diseases. The pro-inflammatory property of rCRT is partially attributable to its ability to self-oligomerize in solution [8], which is supported by the fact that native CRT (nCRT) isolated from mouse liver exists in monomeric form in solution and is much less active compared with prokaryotically expressed rCRT. There are three Cys in CRT. Cys105 and Cys137 form intramolecular disulfide bonds, while Cys163 is free. Jorgensen et al documented that shielding of the free Cys163 in the $\mathrm{N}$ domain is the main reason that $\mathrm{nCRT}$ exists mainly in monomeric form under physiological conditions. Under partial unfolding conditions such as high temperature or low $\mathrm{PH}$, however, the free Cys could be exposed and subsequently help CRT oligomerization [11]. Ironically, however, oligomerization of nCRT, induced by high temperature and/or low $\mathrm{pH}$, did not lead to significantly enhanced macrophage-stimulatory activity of the molecule [12].

Given that rCRT differs from nCRT in its lack of glycosylation, we asked if aberrant glycosylation of eukaryotically expressed CRT (eCRT) would significantly enhance its immunological activity. It has been documented that murine CRT possesses one $\mathrm{N}$-glycosylation site at residue Asn $327[13,14]$ and also that CRT glycosylation is susceptible to modulation by extracellular stimuli $[15,16]$. Additionally, nCRT purified from various tissues carries different glycosylation moieties $[13,14]$. This study was thus designed to investigate if aberrant glycosylation of eukaryotically expressed CRT (eCRT) could augment its immunological activity. Given that aberrant glycosylation of proteins is a hallmark of cancer cells and also correlated to autoimmune diseases in humans, results from this study may provide useful clues for a better understanding of the dysregulated glycosylation of CRT in tumorigenesis and autoimmunity.

\section{Results}

\subsection{Expression, Purification and Functional Characterization of eCRT}

Murine rCRT and rCRT/39-272, both expressed in E. coli, and nCRT from mouse liver were affinity-purified by $\mathrm{Ni}^{+}$columns and ion-exchange columns, respectively, as previously documented [7,8]. Murine CRT gene encoding aa residues 18-412 (less the leader and ER retention sequences) was cloned into expression vector PIRES2 that encodes a secretion leader sequence from pDisplay at the $\mathrm{N}$ terminus and 6-histidine (His) tag at the $\mathrm{C}$ terminus. The resultant plasmid (pIRES2-eCRT) was transfected into $\mathrm{CHO}$ cells for preparation of a cell line (CHO-CRT) stably expressing secreted eCRT, which is affinity-purifiable from the cell culture supernatant by $\mathrm{Ni}^{+}$columns. The purity of fractionated rCRT/39-272, rCRT, nCRT and eCRT was approximately $90 \%$ as judged by Coomassie brilliant blue-stained SDS-PAGE, and the protein bands of expected molecular mass were specifically recognized by rabbit anti-mouse CRT polyclonal Abs in Western blotting (Figure 1A,B). Note that partial degradation occurred to rCRT during the purification process (Figure 1A, lane 2), which is in line with our earlier report [8]. The smaller fragment represented rCRT less the C-terminal 26 residues and it was equally active as full-length rCRT in terms of stimulatory effect against macrophages in vitro and immunogenicity in vivo [8]. Both rCRT/39-272 and rCRT showed strong ability to elicit TNF- $\alpha$ and nitric oxide (NO) production by murine macrophages, while eCRT and nCRT were approximately 10 folds less effective than rCRT and rCRT/39-272 in parallel experiments (Figure 1C,D). 
A

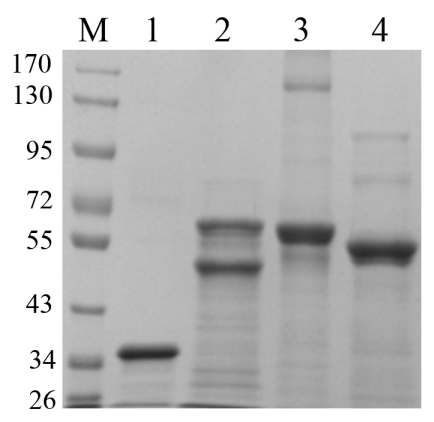

C

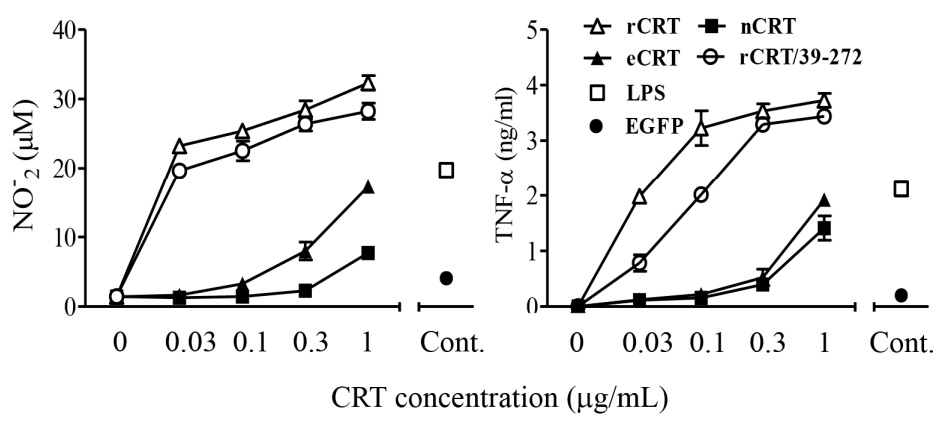

B

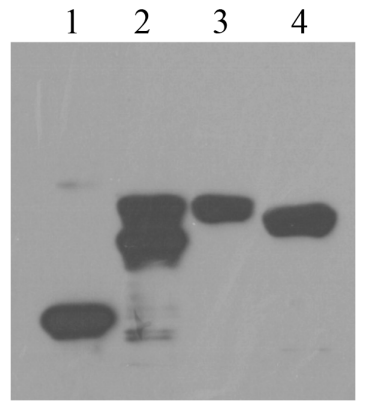

D and nCRT (lanes 1-4, respectively) were separated by SDS-PAGE (A) and detected by rabbit anti-CRT polyclonal antibodies in Western-blot (B). Mouse peritoneal macrophages were stimulated with these CRT preparations of increasing concentrations for $24 \mathrm{~h}$, followed by quantitation of NO (C) and TNF- $\alpha$ (D) in the culture supernatant using Griess Reagent or ELISA, respectively. Macrophages stimulated with LPS $(1 \mathrm{ng} / \mathrm{mL})$ or $\mathrm{rEGFP}(1 \mu \mathrm{g} / \mathrm{mL})$ were included as controls.

\subsection{Immunogenicity of eCRT, rCRT and $n C R T$}

We next compared the ability of eCRT, nCRT and rCRT to induce antibody responses in mice. To avoid interference of residual contaminants in the antigen preparations, eCRT, nCRT and rCRT were run in SDS-PAGE gels, then the dominant protein bands of expected molecular mass were cutout and the resultant gel slices used as immunogens in subsequent animal immunization experiments. Groups of C57BL/ 6 mice were subcutaneously immunized with the antigens, followed by collection of serum samples 10 days thereafter for titration of CRT-specific IgG in ELISAs. Figure 2 shows that antisera from mice immunized with rCRT strongly recognized rCRT, eCRT as well as nCRT. Antisera from eCRT-immunized mice were also able to bind these CRT preparations, albeit with approximately 2-10 folds lower titers compared with that of rCRT-induced antisera. Serum samples from nCRT-immunized, or unimmunized, mice did not show any measurable binding to CRTs, and none of the antisera from CRT-immunized mice recognized recombinant green fluorescence protein (rEGFP) expressed in E. coli (Figure 2). 

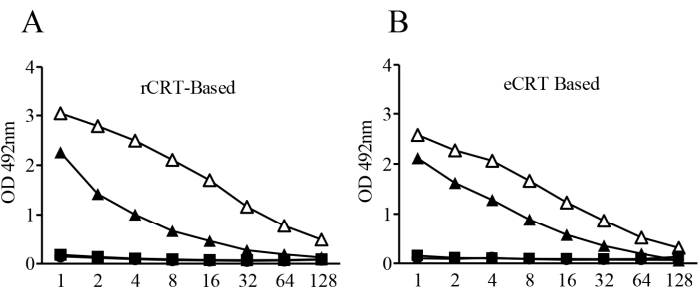

$\mathrm{C}$

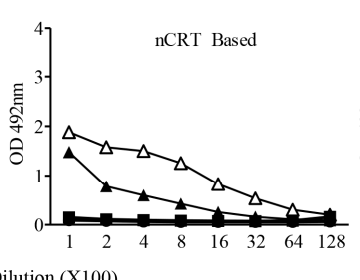

$\mathrm{D}$

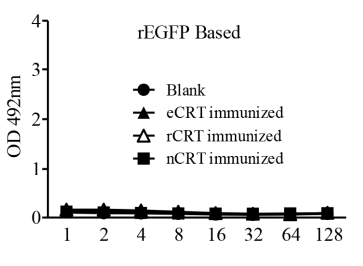

Figure 2. Immunogenicity of eCRT, nCRT and rCRT in mice. SDS-PAGE gel slices containing bands of rCRT, eCRT and nCRT were used as antigens for s.c. immunization of C57/BL6 mice (100 $\mu \mathrm{g} / \mathrm{mouse}$, $n=5$ ) and boosted with $50 \mu \mathrm{g}$ of the same antigen preparations a fortnight later, with blank gel slices as negative control (Blank). The mice were bled 10 days thereafter for sera that were assayed, in triplicate wells, in ELISAs based on rCRT (A), eCRT (B), nCRT (C) or rEGFP (D). The detection Ab was HRP-conjugated goat-anti-mouse IgG with OPD as substrate, and the results are expressed as mean OD492 $\mathrm{nm} \pm \mathrm{SD}$ of three replicates. These are representatives of three independent experiments using proteins from different bulks of purifications.

\subsection{Biochemical Comparison of eCRT and $n C R T$}

Based on our previous results that self-oligomerization could significantly enhance immunological activity of CRT [8], it was reasonable to ask if the enhanced immunogenicity of eCRT over nCRT was due to self-oligomerazation in solution. Native PAGE followed by Western blotting was performed to compare the oligomerization status of the CRT preparations. As expected, rCRT and rCRT/39-272 existed mostly (approximately over $90 \%$ and $60 \%$, respectively) as oligomers in solution at neutral $\mathrm{pH}$. However, eCRT and nCRT existed only in monomeric form in the same conditions (Figure 3A,B). It has previously been documented that calcium depletion as well as $\mathrm{N}$ - and $\mathrm{C}$-terminal truncations promotes CRT oligomerization [17]. Since rCRT and eCRT share the same N- and C-terminal truncations and all CRT preparations were stocked in calcium-free solution, our results imply that glycosylation status may play an important role in controlling self-oligomerization of CRT.

To semi-quantitatively assess the glycosylation levels of CRT preparations, we employed biotinylated ConA, which can bind protein-conjugated oligosaccharide moieties with high affinity, as a detecting agent. ELISA plates were pre-coated with nCRT, eCRT, rCRT or rCRT/39-272, followed by biotin-ConA and then streptavidin-HRP with OPD as substrate. As shown in Figure 3C, ConA binding to eCRT was similarly strong as to nCRT, but there was hardly any ConA binding to rCRT and rCRT/39-272. Even CRT protein coating across the groups was confirmed by strong and specific binding of anti-CRT Abs to all pre-coated wells (Figure 3D). Clearly, relative glycosylation ratios in eukaryotical CRT (eCRT and nCRT) were significantly higher than that in the prokaryotical CRT (rCRT and rCRT/39-272) (Figure 3E). 
A

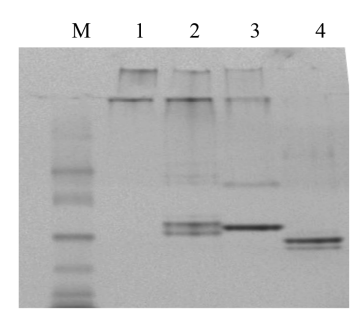

B

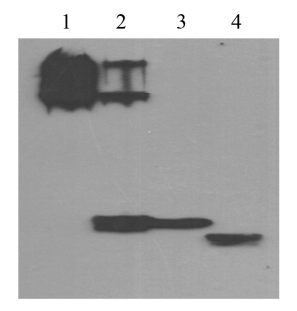

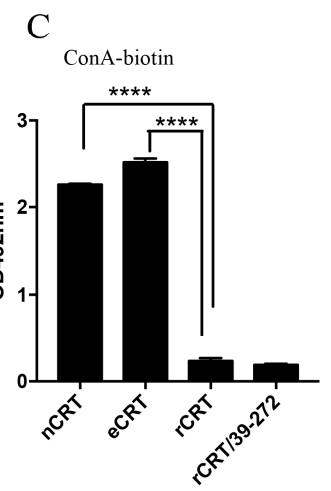
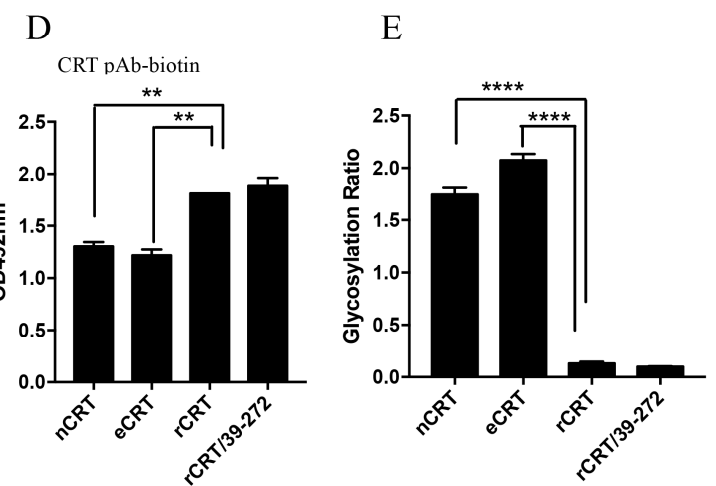

Figure 3. Biochemical properties of eCRT and rCRT. Samples of rCRT/39-272, rCRT, eCRT and nCRT (lanes 1-4, respectively) were compared in Coomassie brilliant blue-stained native PAGE (A), followed by WB using rabbit anti-CRT polyclonal antibody for detection (B). The secondary Ab was HRP labeled goat-anti-rabbit IgG, with OPD as substrate. ELISA plates were pre-coated with nCRT, eCRT, rCRT or rCRT/39-272 followed by ELISAs using ConA-biotin (C) or biotin-conjugated mouse anti-CRT polyclonal antibody (D) for detection. The glycosylation ratios of the CRT preparations were calculated as $\mathrm{OD}_{\mathrm{ConA}} / \mathrm{OD}_{\mathrm{pAb}}(\mathrm{E})$. Results are expressed as mean $\pm \mathrm{SD}$ of three replicates. ${ }^{* * *} p<0.0001 ;{ }^{* *} p<0.01$. These are representatives of 3 independent experiments.

\subsection{Dys-glycosylation of eCRT Correlates to Enhanced Immunological Activity}

In order to further investigate the effect of aberrant glycosylation on the immunological activity of eCRT, tunicamycin, an $\mathrm{N}$-glycosyltransferase inhibitor [18], was employed to treat CHO-CRT cells for preparation of dys-glycosylated eCRT (tun-eCRT). The molecular mass of affinity purified tun-eCRT was slightly lower than eCRT as judged by SDS-PAGE (Figure 4A). The native-PAGE results confirmed that, similar to rCRT, tun-eCRT exists mainly in monomeric rather than oligomeric form. ConA ELISAs further revealed that the level of glycosylation of tun-eCRT was significantly lower than that of eCRT but much higher than that of rCRT (Figure 4B-D). Note that rabbit anti-mouse CRT polycolnal antibodies could not distinguish eCRT from tun-eCRT (Figure 4C), implying that aberrant glycosylation of tun-eCRT did not drastically affect its antigenicity. More importantly, tun-eCRT was apparently more effective than eCRT and nCRT in eliciting macrophage production of NO, TNF- $\alpha$ and IL-6 in vitro (Figure 4E-G). Antisera from mice immunized with tun-eCRT showed significantly higher IgG titers against CRT in ELISAs (Figure 5). Together these results show that aberrant glycosylation enhances pro-inflammatory activity and immunogenicity without affecting monomeric status of eCRT. 

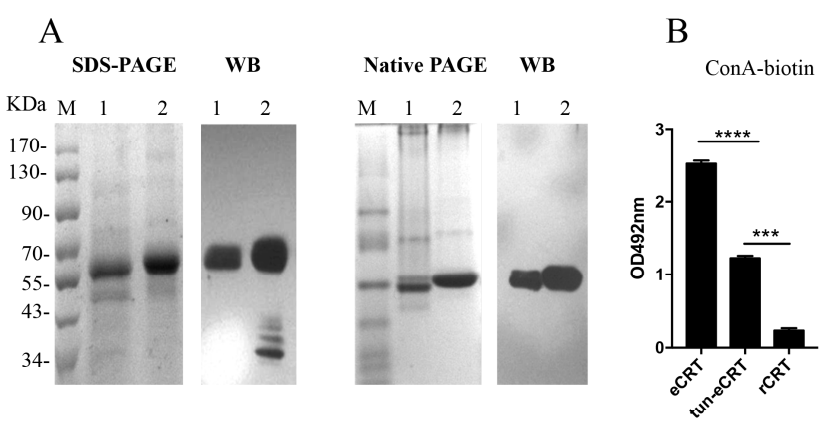
C CRT pAb-biotin

$\mathrm{D}$
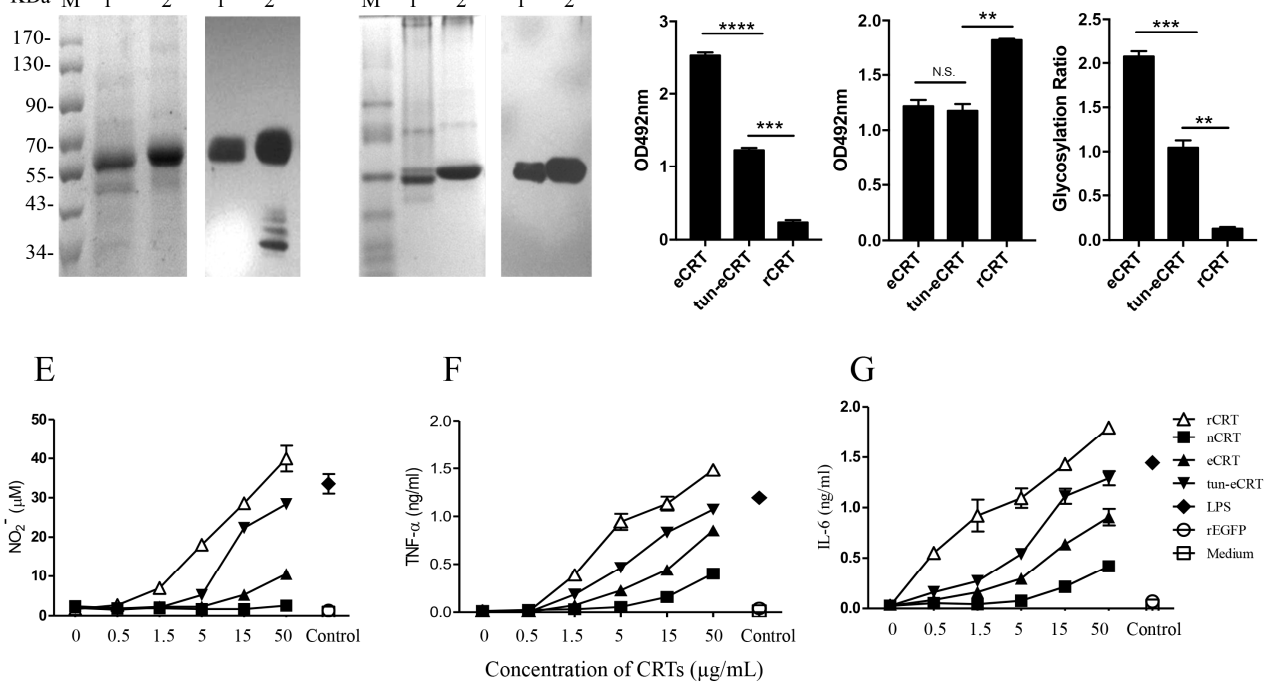

Figure 4. The effect of de-glycosylation on the immune-stimulatory effect of eCRT. Confluent CHO-CRT cells were cultured in serum-free medium in the presence of $5 \mu \mathrm{g} / \mathrm{mL}$ tunicamycin for $48 \mathrm{~h}$. The supernatant was then harvested for purification of tun-eCRT using Ni-columns. Samples of tun-eCRT (lane 1) and eCRT (lane 2) were separated by SDS-PAGE or native PAGE and detected by WB using rabbit anti-CRT polyclonal antibody (A). The glycosylation levels of eCRT, tun-eCRT and rCRT were compared in biotin-ConA binding assays (B) with CRT-Based ELISAs as even protein coating control (C). The glycosylation ratios of the CRT preparations were determined by $\mathrm{OD}_{\mathrm{ConA}} / \mathrm{OD}_{\mathrm{pAb}}(\mathbf{D})$. Mouse peritoneal macrophages were stimulated with increasing concentrations of eCRT, tun-eCRT, nCRT or rCRT for $24 \mathrm{~h}, \mathrm{NO}(\mathrm{E}), \mathrm{TNF}-\alpha(\mathrm{F})$ and IL-6 (G) in the culture supernatant were quantitated by Griess Reagent and ELISAs, respectively. Results are expressed as mean $\pm \mathrm{SD}$ of three replicates. ${ }^{* * * *} p<0.0001 ;{ }^{* * *} p<0.001{ }^{* *} p<0.01$. These are representatives of three independent experiments.

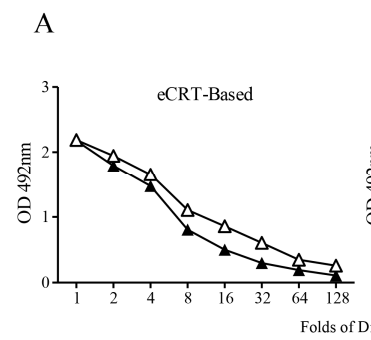

\section{B}

C

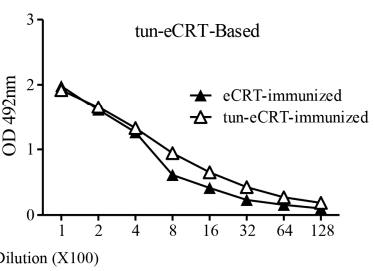

$\mathrm{D}$
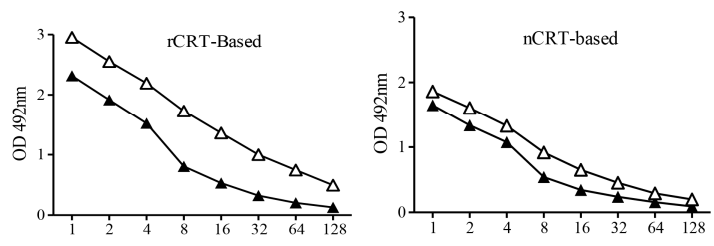

Folds of Dilution (X100)

Figure 5. Comparison of immunogenicity of eCRT and tun-eCRT. Coomassie-blue stained SDS-PAGE gel slices containing eCRT or tun-eCRT were used immunogens for s.c. immunization of C57/BL6 mice $(n=5)$. Immunized mice were boosted with the same antigens with fortnight intervals. Mice were bled 10 days thereafter for detection of antigen-specific IgG in ELISAs based on eCRT (A), tun-eCRT (B), rCRT (C) or nCRT (D). Results are expressed as mean \pm SD of three replicates. These are representatives of 3 independent experiments. 


\section{Discussion}

CRT is susceptible to various post-translation modifications with important functional consequences. It has been reported that CRT can be phosphorylated by PKC [19] or c-src [20], which enables the molecule to stabilize mRNA in the cell. Arginylation of CRT, which causes a greater degree of dimerization and oligomerization of the molecule [21], makes the cell more susceptible to apoptosis [22]. Glycosylation is another important post-translation modification of CRT, which can impact its cellular redistribution [23]. CRT glycosylation changes rapidly and dynamically in response to extracellular stimuli such as heat shock and cold stress $[15,24]$. PKC activation could also abolish CRT N-glycosylation but increase $O$-linked- $N$-acetylglucosamine modification [25]. Furthermore, nCRT isolated from different sources, such as rat and bovine liver [26,27], bovine brain [28], hamster ovary [15], or human myeloid cells [29] appears to possess diverse glycosylation moieties. However, contribution of the glycosylation moieties of CRT to its oligomerization under different conditions has not been thoroughly investigated.

Here we compared eCRT and tun-eCRT for glycosylation level, self-oligomerization, macrophage-stimulatory activity in vitro and also immunogenicity in vivo. Our results indicate that tunicamycin-induced dys-glycosylation of eCRT significantly enhanced the immune-stimulatory activity and immunogenicity without affecting the monomeric status of the molecule. The lack of immunogenicity of nCRT in mice can be explained by self-tolerance of the host to tissue antigens. Yet eCRT is identical to nCRT in terms of amino acid sequence, the apparently stronger immunogenicity of eCRT than nCRT has to be explained by either incorrect folding, increased self-oligomerization or aberrant post-translation modifications such as glycosylation. Although the extraordinarily strong immune-stimulatory activity of prokaryotically expressed rCRT was attributable to its self-oligomerization in solution [8], eCRT as well as tun-eCRT did not seem to self-oligomerize in solution (Figure 3), thereby arguing against any substantial role of oligomerization in licensing potent immunological activity to eukaryotical CRT. We propose that dys-glycosylation rather than self-oligomerization is a more important factor rendering eCRT immunological activities. Although the tunicamycin could inhibit glycosylation of recombinant CRT in CHO, tunicamycin treatment is not for thoroughly de-glycosylation and might induce other effects on CHO cells thus affecting CRT post-translational modification. An alternative system for preparation of de-glycosylated CRT would be $\mathrm{CHO}$-Lec1 cells that are deficient in N-acetylglucosaminyltransferase I (GlcNAc-TI) and do not synthesize $N$-glycans. The only possible site for glycosylation of murin CRT is Asn 327, thus murine CRT with amino acid 327 mutation could also be used for confirmation the contribution of glycosylation to immune-stimulatory activity of CRT. Both lines of work are currently in progress in this laboratory.

Higher levels of serum CRT positively correlate with RA, SLE and certain cancers in humans [1-4,7]. Ding et al. also reported that extracellular CRT may promote angiogenesis via a NO-mediated pathway in RA [6]. This is in line with our finding that tun-eCRT is able to elicit inflammatory mediator (including NO) production by macrophages. Aberrant protein glycosylation has been associated with tumors as well as various autoimmune syndromes, such as RA and SLE [30]. Defect in protein N-glycosylation pathway is a genetic cause of autoimmune disease [31]. A bioinformatical approach by Szabo and colleagues predicated substantial impact of aberrant glycosylation of certain proteins on $\mathrm{T}$ cell autoimmunity [32]. These data point to a possibility that CRT from patients may possess potent pro-inflammatory (immunostimulatory) or angiogenesis activities as does tun-eCRT. Therefore, the glycosylation and immunological characteristics of CRT in patient sera or in cancerous tissues merits further detailed investigation. 


\section{Materials and Methods}

\subsection{Construction of Plasmid pIRES-eCRT}

Total cellular RNA was extracted from mouse tumor cell line EL4 (ATCC) using TRIzol (Invitrogen, Carlsbad, CA, USA) and then reverse transcribed with Oligo-dT using a SuperScript II reverse transcriptase kit (Invitrogen, Carlsbad, CA, USA) according to the manufacturer's instructions. The resultant cDNA was employed as template for amplification of the gene encoding CRT fragment 18-412 by PCR using forward primer 5'-ATTCCGCGGTGACCCTGCCATCTATTTCAAA-3' with a Sac II restriction site and reverse primer 5'-ATATAGTCGACCTAGTGGTGGTGGTGGTGGTGGTGGTTGGCCAGGGGATTCTTCC-3' with a Sal I restriction site. The PCR product (encoding the target sequence with a C-terminal 6X polyhistidine tag) was cloned into expression vector pDisplay (Invitrogen, Carlsbad, CA, USA). The gene encoding CRT/18-242 with N-terminus leader sequence of pDisplay and C-terminus His tag was finally cloned into expression vector pIRES2 using a Xho I and a Sal I restriction sites.

\subsection{Expression and Purification of CRT}

For preparation of secreted eCRT, $\mathrm{CHO}$ cells were transfected with plasmid pIRES-eCRT using lipofectamine 2000 (Invitrogen, Carlsbad, CA, USA) according to the manufacturer's specifications. Stable transfections (CHO-eCRT) were selected in G418 (1 mg/mL, Sigma, St. Louis, MO, USA) for two weeks. Transfected $\mathrm{CHO}$ cells were grown in $10 \mathrm{~cm}$ dishes until confluent, culture medium was then removed and changed into FBS-free DMEM (Hyclone, Logan, UT, USA) after being washed with warm PBS. Cells were further incubated for $48 \mathrm{~h}\left(5 \% \mathrm{CO}_{2}, 37^{\circ} \mathrm{C}\right)$ and supernatant harvested and depleted cell debris by centrifugation, followed by clearing using $0.45 \mu \mathrm{M}$ filter (Millipore, Billerica, MA, USA). For preparation of tun-eCRT, CHO-CRT cells were cultured in FBS-free DMEM in the presence of tunicamycine (5 $\mathrm{g} / \mathrm{mL}$, Sigma, St. Louis, MO, USA) for $48 \mathrm{~h}$. The secreted recombinant eCRT was purified from the supernatant using Ni-column (GE, Little Chalfont, Buckinghamshire, UK) following the manufacturers' instructions. Briefly, the supernatant was loaded on the column, washed with buffer containing $20 \mathrm{mM}$ Tris- $\mathrm{HCl}, 0.5 \mathrm{M} \mathrm{NaCl}, 20 \mathrm{mM}$ Imidazole, $\mathrm{pH}$ 7.9. The proteins were eluted with wash buffer containing $60 \mathrm{mM}$ Imidazole and dialyzed against PBS, pH 7.9, for 16 h. Prokaryotical rCRT and rEGFP were expressed and purified as previously described [7,8]. Briefly, E. coli BL21 (DE3) (Stratagene, La Jolla, CA, USA) containing PET28a-EGFP or PET28a-CRT were grown to log phage at cell concentration of OD600 was $0.6-0.8$ in medium with $30 \mu \mathrm{g} / \mathrm{mL}$ kanamycin at $37^{\circ} \mathrm{C}$, and protein expression was induced by adding $0.1 \mathrm{mmol} / \mathrm{L}$ isopropylthio- $\beta$-D-galactoside (IPTG) in LB medium for further incubation at $30^{\circ} \mathrm{C}$ for $5 \mathrm{~h}$. The bacterial lysate (with protease inhibitor PMSF) was loaded on the column, washed with buffer containing $20 \mathrm{mM}$ Tris- $\mathrm{HCl}, 0.5 \mathrm{M} \mathrm{NaCl}, 20 \mathrm{mM}$ Imidazole, pH 7.9. The proteins were eluted with wash buffer containing $300 \mathrm{mM}$ Imidazole. nCRT was purified as previously described using ion-exchange column [8]. All the proteins were desalted into PBS and concentrated by ultra-centrifugate tube. Concentration of protein was quantified using Pierce BCA Protein Assay Kit (Pierce, Invitrogen). The proteins were treated by polymyxin B-agarose (GE Health, Little Chalfont, Buckinghamshire, UK) for three times before being used and endotoxin acitivity of proteins were both less than $0.01 \mathrm{EU} / \mu \mathrm{g}$ protein assessed by the limulus amebocyte assay.

\subsection{SDS-PAGE, Native PAGE and Western Blotting}

For SDS-PAGE, loading buffer with 1\% SDS and 2-ME was used. For native PAGE, the loading buffer did not contain any detergent or reducing agents. The gels were stained with Coomassie brilliant blue or transferred to PVDF membranes (Millipore, Billerica, MA, USA). Immunoblots were blocked with $5 \%$ non-fat milk $(w / v)$ in TBS buffer for $1 \mathrm{~h}$ at room temperature then incubated with primary rabbit antibody against CRT (Pierce, 1:1000) over-night. Immunoreactive bands were detected using HRP-conjugated secondary antibodies with the western lighting chemiluminescence plus reagent (BD Pharmingen, San Jose, CA, USA). 


\subsection{Isolation and Culture of Mouse Peritoneal Macrophages}

For preparation of murine macrophages, C57BL/6 mice (female, 6-8 weeks) were injected intraperitoneally with $1 \mathrm{~mL}$ 3\% thioglycollate (BD Pharmingen, San Jose, CA, USA). Peritoneal cells were harvested by peritoneal lavage with RPMI-1640 $72 \mathrm{~h}$ after injection and seeded in 96-well tissue culture plates. After removing non-adherent cells, the remaining cells were $90 \%$ positive for macrophage surface marker F4/80, as determined by FACS analysis. Macrophages were cultured in complete R10 medium: RPMI-1640 supplemented with 10\% $(v / v)$ fetal bovine serum (Hyclone, Logan, UT, USA), penicillin/streptomycin $(100 \mathrm{U} / \mathrm{mL})$, L-glutamine $(2 \mathrm{mM})$, and $\beta$-ME $\left(5 \times 10^{-5} \mathrm{M}\right)$. After stimulation with eCRT, tun-eCRT, rCRT and EGFP for $24 \mathrm{~h}$, TNF- $\alpha$ and IL-6 in supernatant was determined by ELISA kit (Biolegend, San Diego, CA, USA) according to manufacturer's instructions. NO in supernatant was determined by NO detection Kit (Promega, Fitchburg, WI, USA). All procedures were approved by Ethic Committee of Soochow University (IACUC-01326)

\subsection{Immunization of Mice and Protein Based ELISA}

CRT preparations (100 $\mu$ g per gel) were separated in SDS-PAGE 10\% gels, gel slices containing expected CRT bands were cutout with a razorblade and then frozen in liquid nitrogen, minced and then re-suspended in PBS. Groups of C57BL/ 6 mice (5 per group) were subcutaneously immunized with $100 \mu \mathrm{L}$ CRT and boosted twice a fortnight later interval with the same antigens. The immunized mice were bled 7 days after the immunization for assessment of serum titers against plate-coated CRT in ELISAs.

For CRT-based ELISAs, $2 \mu \mathrm{g} / \mathrm{mL}$ protein was coated on plate in carbonate buffer ( $\mathrm{pH} 9.6)$ and subsequently incubated with blocking solution ( $2 \%$ BSA in PBS) for $2 \mathrm{~h}$ at $37^{\circ} \mathrm{C}$. The wells were washed five times with PBS containing 0.05\% Tween 20 (PBS-T) and then $100 \mu \mathrm{L}$ of diluted immunized mouse sera in PBS were added in triplicates and incubated for $2 \mathrm{~h}$ at $37^{\circ} \mathrm{C}$. After five washes with PBS-T, the plates were incubated with HRP-labeled goat anti-mouse IgM or IgG Abs (Southern Biotech) for $1 \mathrm{~h}$ at $37^{\circ} \mathrm{C}$. The reaction was developed with $100 \mu \mathrm{L}$ of $\mathrm{O}$-phenylenediamine (OPD) (Sigma-Aldrich, St. Louis, MO, USA) for $5 \mathrm{~min}$ and stopped with $50 \mu \mathrm{L}$ of $2 \mathrm{M} \mathrm{H}_{2} \mathrm{SO}_{4}$. OD was measured at $492 \mathrm{~nm}$ in an ELISA spectrophotometer (Bio-Tek, Winooski, VT, USA).

\subsection{Detection of $N$-glycosylation in CRTs}

Lectin blot analysis was essentially the same as ELISAs as previously described [25]. ELISA plates were coated with CRTs $(0.2 \mu \mathrm{g} / \mathrm{mL}$ ) in carbonate buffer ( $\mathrm{pH} 9.6)$ and subsequently incubated with blocking solution ( $2 \%$ BSA in PBS, $w / v)$ for $2 \mathrm{~h}$ at $37^{\circ} \mathrm{C}$. The wells were washed five times with PBS-T and then incubated with $1 \mu \mathrm{g} / \mathrm{mL}$ biotinylated Con A (Sigma, St. Louis, MO, USA) for $1 \mathrm{~h}$ at $37^{\circ} \mathrm{C}$. N-glycosylated proteins were visualized by incubation with HRP-conjugated streptavidin and developed with $100 \mu \mathrm{L}$ of O-phenylenediamine (OPD) (Sigma-Aldrich, St. Louis, MO, USA) for 5 min and stopped with $50 \mu \mathrm{L}$ of $2 \mathrm{M} \mathrm{H}_{2} \mathrm{SO}_{4}$. OD was measured at $492 \mathrm{~nm}$ in an ELISA spectrophotometer. No detectable ConA binding was observed when Peptide N-glycosidase F (PNGase F)-treated eCRT and tun-eCRT were analyzed in this system. Negative controls were also obtained by incubating wells with biotinylated ConA in the presence of $300 \mathrm{mM}$ free glucose prior to visualization with HRP-conjugated streptavidin.

\subsection{Statistical Analysis}

All experiments were repeated at least three times. Statistical analysis was performed using the independent samples $t$ test or one-way ANOVA and Tukey's post hoc tests. Differences were considered statistically significant at $p<0.05$. 
Acknowledgments: This study was supported by grants from the Program for Changjiang Scholars and Innovative Research Team (IRT1075) and National Foundation of Nature Science (31370908/31570868) and also Foundation for Application and Fundamental Research of Healthcare (SYS201667) of China.

Author Contributions: F.-Y.G. and X.-M.G. designed the conception of this study. F.-Y.G., Z.G., C.-C.D., C.H., J.W. prepared and carried out the experiments. The statistical analyses were implemented by F.-Y.G. and J.W. Drafting of manuscript was carried out by F.-Y.G. and X.-M.G.

Conflicts of Interest: The authors declare no conflict of interest. The founding sponsors had no role in the design of the study; in the collection, analyses, or interpretation of data; in the writing of the manuscript, and in the decision to publish the results.

\section{References}

1. Zamanian, M.; Veerakumarasivam, A.; Abdullah, S.; Rosli, R. Calreticulin and cancer. Pathol. Oncol. Res. 2013, 19, 149-154. [CrossRef] [PubMed]

2. Sollazzo, D.; Forte, D.; Polverelli, N.; Perricone, M.; Romano, M.; Luatti, S.; Vianelli, N.; Cavo, M.; Palandri, F.; Catani, L. Circulating calreticulin is increased in myelofibrosis: Correlation with interleukin-6 plasma levels, bone marrow fibrosis, and splenomegaly. Mediat. Inflamm. 2016, 2016, 5860657. [CrossRef] [PubMed]

3. Kageyama, S.; Isono, T.; Matsuda, S.; Ushio, Y.; Satomura, S.; Terai, A.; Arai, Y.; Kawakita, M.; Okada, Y.; Yoshiki, T. Urinary calreticulin in the diagnosis of bladder urothelial carcinoma. Int. J. Urol. 2009, 16, 481-486. [CrossRef] [PubMed]

4. Liu, R.; Gong, J.; Chen, J.; Li, Q.; Song, C.; Zhang, J.; Li, Y.; Liu, Z.; Dong, Y.; Chen, L.; et al. Calreticulin as a potential diagnostic biomarker for lung cancer. Cancer Immunol. Immunother. 2012, 61, 855-864. [CrossRef] [PubMed]

5. Pike, S.E.; Yao, L.; Jones, K.D.; Cherney, B.; Appella, E.; Sakaguchi, K.; Nakhasi, H.; Teruya-Feldstein, J.; Wirth, P.; Gupta, G.; et al. Vasostatin, a calreticulin fragment, inhibits angiogenesis and suppresses tumor growth. J. Exp. Med. 1998, 188, 2349-2356. [CrossRef] [PubMed]

6. Ding, H.; Hong, C.; Wang, Y.; Liu, J.; Zhang, N.; Shen, C.; Wei, W.; Zheng, F. Calreticulin promotes angiogenesis via activating nitric oxide signalling pathway in rheumatoid arthritis. Clin. Exp. Immunol. 2014, 178, 236-244. [CrossRef] [PubMed]

7. Hong, C.; Qiu, X.; Li, Y.; Huang, Q.; Zhong, Z.; Zhang, Y.; Liu, X.; Sun, L.; Lv, P.; Gao, X.M. Functional analysis of recombinant calreticulin fragment 39-272: Implications for immunobiological activities of calreticulin in health and disease. J. Immunol. 2010, 185, 4561-4569. [CrossRef] [PubMed]

8. Huang, S.H.; Zhao, L.X.; Hong, C.; Duo, C.C.; Guo, B.N.; Zhang, L.J.; Gong, Z.; Xiong, S.D.; Gong, F.Y.; Gao, X.M. Self-oligomerization is essential for enhanced immunological activities of soluble recombinant calreticulin. PLoS ONE 2013, 8, e64951. [CrossRef] [PubMed]

9. Dayer, J.M.; Choy, E. Therapeutic targets in rheumatoid arthritis: The interleukin-6 receptor. Rheumatology 2010, 49, 15-24. [CrossRef] [PubMed]

10. McInnes, I.B.; Schett, G. The pathogenesis of rheumatoid arthritis. N. Engl. J. Med. 2011, 365, $2205-2219$. [CrossRef] [PubMed]

11. Jorgensen, C.S.; Ryder, L.R.; Steino, A.; Hojrup, P.; Hansen, J.; Beyer, N.H.; Heegaard, N.H.; Houen, G. Dimerization and oligomerization of the chaperone calreticulin. Eur. J. Biochem. 2003, 270, 4140-4148. [CrossRef] [PubMed]

12. He, M.C.; Wang, J.; Wu, J.; Gong, F.Y.; Hong, C.; Xia, Y.; Zhang, L.J.; Bao, W.R.; Gao, X.M. Immunological activity difference between native calreticulin monomers and oligomers. PLOS ONE 2014, 9, e105502. [CrossRef] [PubMed]

13. Michalak, M.; Corbett, E.F.; Mesaeli, N.; Nakamura, K.; Opas, M. Calreticulin: One protein, one gene, many functions. Biochem. J. 1999, 344, 281-292. [CrossRef] [PubMed]

14. Yokoyama, M.; Hirata, K. New function of calreticulin: Calreticulin-dependent mrna destabilization. Circ. Res. 2005, 97, 961-963. [CrossRef] [PubMed]

15. Jethmalani, S.M.; Henle, K.J.; Kaushal, G.P. Heat shock-induced prompt glycosylation. Identification of p-sg67 as calreticulin. J. Biol. Chem. 1994, 269, 23603-23609. [PubMed]

16. Jeffery, E.; Peters, L.R.; Raghavan, M. The polypeptide binding conformation of calreticulin facilitates its cell-surface expression under conditions of endoplasmic reticulum stress. J. Biol. Chem. 2011, 286, 2402-2415. [CrossRef] [PubMed] 
17. Rizvi, S.M.; Mancino, L.; Thammavongsa, V.; Cantley, R.L.; Raghavan, M. A polypeptide binding conformation of calreticulin is induced by heat shock, calcium depletion, or by deletion of the c-terminal acidic region. Mol. Cell 2004, 15, 913-923. [CrossRef] [PubMed]

18. Heifetz, A.; Keenan, R.W.; Elbein, A.D. Mechanism of action of tunicamycin on the udp-glcnac: Dolichyl-phosphate glc-nac-1-phosphate transferase. Biochemistry 1979, 18, 2186-2192. [CrossRef] [PubMed]

19. Rendon-Huerta, E.; Mendoza-Hernandez, G.; Robles-Flores, M. Characterization of calreticulin as a protein interacting with protein kinase c. Biochem. J. 1999, 344, 469-475. [CrossRef] [PubMed]

20. Mueller, C.F.; Wassmann, K.; Berger, A.; Holz, S.; Wassmann, S.; Nickenig, G. Differential phosphorylation of calreticulin affects at1 receptor mrna stability in vsmc. Biochem. Biophys. Res. Commun. 2008, 370, 669-674. [CrossRef] [PubMed]

21. Carpio, M.A.; Decca, M.B.; Lopez Sambrooks, C.; Durand, E.S.; Montich, G.G.; Hallak, M.E. Calreticulin-dimerization induced by post-translational arginylation is critical for stress granules scaffolding. Int. J. Biochem. Cell Biol. 2013, 45, 1223-1235. [CrossRef] [PubMed]

22. Lopez Sambrooks, C.; Carpio, M.A.; Hallak, M.E. Arginylated calreticulin at plasma membrane increases susceptibility of cells to apoptosis. J. Biol. Chem. 2012, 287, 22043-22054. [CrossRef] [PubMed]

23. Jethmalani, S.M.; Henle, K.J.; Gazitt, Y.; Walker, P.D.; Wang, S.Y. Intracellular distribution of heat-induced stress glycoproteins. J. Cell. Biochem. 1997, 66, 98-111. [CrossRef]

24. Komatsu, S.; Yamada, E.; Furukawa, K. Cold stress changes the concanavalin a-positive glycosylation pattern of proteins expressed in the basal parts of rice leaf sheaths. Amino Acids 2009, 36, 115-123. [CrossRef] [PubMed]

25. Cristina Castaneda-Patlan, M.; Razo-Paredes, R.; Carrisoza-Gaytan, R.; Gonzalez-Mariscal, L.; Robles-Flores, M. Protein kinase $\mathrm{c}$ is involved in the regulation of several calreticulin posttranslational modifications. Int. J. Biochem. Cell Biol. 2010, 42, 120-131. [CrossRef] [PubMed]

26. Van, P.N.; Peter, F.; Soling, H.D. Four intracisternal calcium-binding glycoproteins from rat liver microsomes with high affinity for calcium. No indication for calsequestrin-like proteins in inositol 1,4,5-trisphosphate-sensitive calcium sequestering rat liver vesicles. J. Biol. Chem. 1989, 264, 17494-17501. [PubMed]

27. Khanna, N.C.; Tokuda, M.; Waisman, D.M. Comparison of calregulins from vertebrate livers. Biochem. J. 1987, 242, 245-251. [CrossRef] [PubMed]

28. Matsuoka, K.; Seta, K.; Yamakawa, Y.; Okuyama, T.; Shinoda, T.; Isobe, T. Covalent structure of bovine brain calreticulin. Biochem. J. 1994, 298, 435-442. [CrossRef] [PubMed]

29. Denning, G.M.; Leidal, K.G.; Holst, V.A.; Iyer, S.S.; Pearson, D.W.; Clark, J.R.; Nauseef, W.M.; Clark, R.A. Calreticulin biosynthesis and processing in human myeloid cells: Demonstration of signal peptide cleavage and N-glycosylation. Blood 1997, 90, 372-381. [PubMed]

30. Raghav, S.K.; Gupta, B.; Agrawal, C.; Saroha, A.; Das, R.H.; Chaturvedi, V.P.; Das, H.R. Altered expression and glycosylation of plasma proteins in rheumatoid arthritis. Glycoconj. J. 2006, 23, 167-173. [CrossRef] [PubMed]

31. Chui, D.; Sellakumar, G.; Green, R.; Sutton-Smith, M.; McQuistan, T.; Marek, K.; Morris, H.; Dell, A.; Marth, J. Genetic remodeling of protein glycosylation in vivo induces autoimmune disease. Proc. Natl. Acad Sci. USA 2001, 98, 1142-1147. [CrossRef] [PubMed]

32. Szabo, T.G.; Palotai, R.; Antal, P.; Tokatly, I.; Tothfalusi, L.; Lund, O.; Nagy, G.; Falus, A.; Buzas, E.I. Critical role of glycosylation in determining the length and structure of $t$ cell epitopes. Immunome Res. 2009, 5, 4. [CrossRef] [PubMed]

Sample Availability: Samples of the compounds are not available from the authors. 